\title{
Les sciences de la matière en micropesanteur
}

\author{
BERNARD ZAPPOLI ${ }^{\mathrm{a}}$ \\ CNES / Direction des Programmes, Université Paul Sabatier, Toulouse, France
}

Reçu le 29 août 2003, accepté le 19 février 2004

\begin{abstract}
Résumé - Née avec les premiers vols habités, la recherche dans le domaine des sciences de la matière en micropesanteur est pratiquée aujourd'hui par une communauté nationale et internationale qui a appris à utiliser avec pertinence un outil qu'elle n'avait pas demandé et pour la mise en place duquel elle avait peu été consultée. L'étude à des fins scientifiques ou technologiques des phénomènes physiques en micropesanteur est devenue un élément de la recherche spatiale. Après avoir rappelé comment se manifeste l'ambiance de micropesanteur, nous montrons comment au cours des dernières années et pour se limiter à la communauté scientifique française, celle-ci a obtenu des résultats remarqués qu'il aurait été impossible d'obtenir au sol notamment dans le domaine de la solidification d'alliages métalliques et de la physique des fluides au voisinage du point critique. Nous présentons le bilan et les perspectives dans les cinq domaines principaux dans lesquels ont été classés les actions de recherche en micropesanteur pour lesquels nous présentons les éléments les plus significatifs : la solidification et la croissance cristalline, la combustion, les interfaces fluides, les objets lévités et les fluides critiques et supercritiques. Nous rappelons les liens importants qui existent entre ces travaux et la gestion des fluides en orbite. Nous présentons enfin un projet d'expérimentation dans la Station Spatiale Internationale qui est le plus représentatif de ceux qui sont à la fois pertinents et porteurs de résultats de premier plan, la formation des structures lors de la croissance d'alliages eutectiques modèles transparents.
\end{abstract}

Mots clés : Matière condensée / microgravité / combustion / interface / phénomènes critiques

\begin{abstract}
Condensed matter physics under microgravity conditions. A national and international community is involved in condensed mater physics research under microgravity conditions. This research was born with the first manned flights and is now a component of space research. This community learned how to perform relevant studies, with a new tool she did not asked for and for the development of which she was not too much consulted. We first show how the microgravity environment manifests itself on condensed matter and how the French scientific community obtained remarkable results which could never have been obtained on the ground, particularly in the field of the solidification of metallic alloys or in the filed of critical point phenomena. We make the assessment and give the perspectives in five domains for which we present the most significant elements: solidification and crystal growth, combustion, interface between fluids, levitated objects and critical and supercritical fluids. We recall the links which exist between all of these work and fluid management in space. We finally present one project witch is the most representative of those charaterized by a strong microgravity relevance and which are bearer of new physics: pattern formation during the solidification of transparent model eutectic alloys.
\end{abstract}

Key words: Condensed matter physics / microgravity / combustion / interface / critical phenomena

\section{Quelques définitions}

La micropesanteur est l'ambiance dynamique, relativement à un repère en translation lié à leur centre de gravité, d'objets matériels se déplaçant dans un champ de gravité sous l'effet seul de leur vitesse initiale. Le poids d'un objet matériel ayant la position du centre de gravité

\footnotetext{
a Auteur correspondant : Bernard.Zappoli@cnes.fr
}

et la masse totale du système, est compensé à tout instant par la force d'inertie d'entraînement. Cette compensation n'est qu'approximative pour les autres objets qui se trouvent en situation de micropesanteur. Dans une station spatiale en orbite autour de la terre ou dans un avion en vol parabolique, les objets situés dans le véhicule sont ainsi en micropesanteur relative, ils n'ont presque plus de poids apparent, celui-ci étant presque équilibré par la force d'inertie d'entraînement (qui est la force centrifuge 


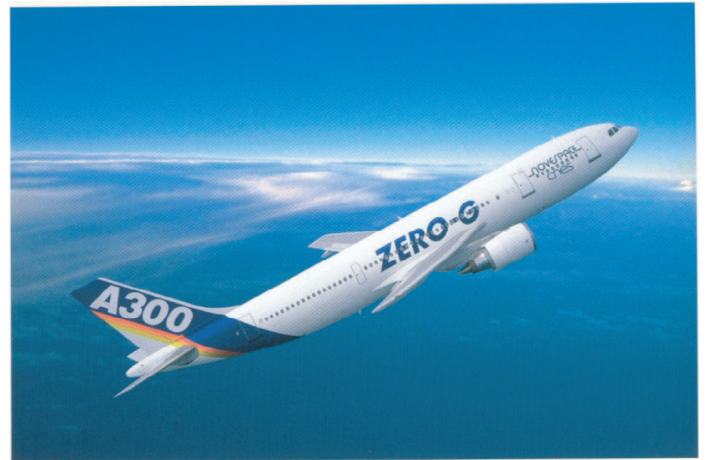

Fig. 1. L'avion A 300 en phase ascensionnelle d'une trajectoire parabolique balistique. Trois séries de trente paraboles de vingt secondes de microgravité chacune constituent une campagne de vols paraboliques. Relativement au centre de masse de l'avion, les objets qu'il contient sont en micropesanteur apparente relative. Leur poids est quasiment équilibré par la force d'inertie d'entraînement.

pour une station orbitale). Seul le centre de masse est donc en pesanteur nulle. On peut noter à ce propos la différence entre pesanteur et gravité nulle : alors que la pesanteur nulle est caractérisée par une résultante nulle des forces de gravité et d'inertie, seule une résultante nulle des forces de gravité caractérise une gravité nulle. On reproduit sur terre la micropesanteur en lâchant des objets depuis des tours dites à chute libre ou dans des puits de mine désaffectés aménagés ou encore en les faisant voler dans des avions en vols paraboliques (Fig. 1).

\section{Comment la suppression de la gravité joue-t-elle sur la matière?}

Pour les objets fluides, la force volumique de gravité qui s'applique à chaque volume élémentaire est, elle aussi, quasiment équilibrée par la force d'inertie et les particules fluides n'ont plus de poids apparent. La micropesanteur a donc une influence sur les phases fluides dans lesquelles elle a des effets statiques comme la suppression de la pression hydrostatique, et des effets dynamiques, comme la suppression de la sédimentation et de la convection. Ces mouvements étant engendrés au sol par les forces dépendant de différences de densité, l'action indirecte de la micropesanteur sera ainsi d'autant plus sensible que celles-ci seront plus importantes. La micropesanteur a donc un effet indirect sur les systèmes physiques comportant au moins une phase fluide présentant de fortes différences de densité. Elle y modifie profondément les conditions de transport de matière et d'énergie ainsi que la morphologie et l'extension spatiale des interfaces entre fluides ou entre un fluide et une autre phase. La micropesanteur permet donc d'étudier dans des conditions de transport purement diffusives des phénomènes physiques qui au sol sont masqués, déformés, ou encore dénaturés, par la présence de mouvements perturbateurs dans les phases fluides. La prise en compte de ces éléments dans la définition du programme scientifique a évité que soient réalisées des expériences de portée limitée, peu imaginatives ou porteuses d'espoirs inconsidérés. Pour plus de détail le lecteur pourra se rapporter à la référence [1].

\section{Qu'est ce que la micropesanteur?}

La micropesanteur est d'abord un outil pour obtenir des connaissances qu'il serait impossible d'acquérir au sol, qu'il s'agisse de recherche scientifique ou d'informations contribuant à améliorer les procédés industriels au sol. Ce n'est pas une discipline scientifique, c'est un grand instrument. D'autre part, comme il est très difficile de créer une gravité artificielle dans l'espace, tout autant que de la supprimer au sol, la micropesanteur est aussi une caractéristique incontournable de l'environnement spatial auquel les technologies de lanceurs et de satellites doivent s'adapter. C'est là le second aspect de la micropesanteur qui apparaît comme une contrainte technologique forte dont les rapports avec la recherche en sciences de la matière sont très étroits et constituent à notre avis un domaine d'applications industrielles très ouvert.

\section{La solidification et la croissance cristalline}

Dans ce domaine, la micropesanteur permet d'étudier les structures qui se forment à l'interface entre un liquide et un solide qui croît à partir du liquide. Il peut s'agir d'alliages métalliques ou de matériaux modèles transparents, ces derniers étant observables par des méthodes optiques tout en donnant des informations sur les mécanismes de solidification des matériaux métalliques sans toutefois en reproduire tous les paramètres comme la différence de conductivité thermique entre le solide et le liquide. De très nombreux travaux ont été conduits par les grandes nations ayant accès à l'espace. Ceux-ci avaient initialement pour but de montrer la rentabilité industrielle de la production spatiale de matériaux aux propriétés nouvelles, à très haute valeur ajoutée par exemple, comme des détecteurs gamma qui sont des semi-conducteurs d'intérêt stratégique. Cette action s'est soldée par un échec au sens où n'ont pu être obtenus au mieux que des échantillons de matériaux de référence, la rentabilité industrielle étant mise en brèche par le coût du transport spatial. En revanche, il est rapidement apparu que la micropesanteur donnait accès à l'observation en régime purement diffusif de la formation de structures à l'interface de solidification et pouvait avoir de ce fait un intérêt scientifique dans le domaine de la solidification lorsque la vitesse de l'interface est importante ou que la phase mère est concentrée. La nécessité de transporter vers l'orbite des masses importantes d'échantillons et de les retourner au sol après avoir été fondus et re-solidifiés dans l'espace a présenté une difficulté logistique importante. Celle-ci, dans certains types d'expériences, a été contournée en utilisant l'effet Seebeck pour accéder à la température de l'interface de solidification et n'avoir plus à transmettre au sol que des signaux électriques. Mise au point au Laboratoire d'Étude 


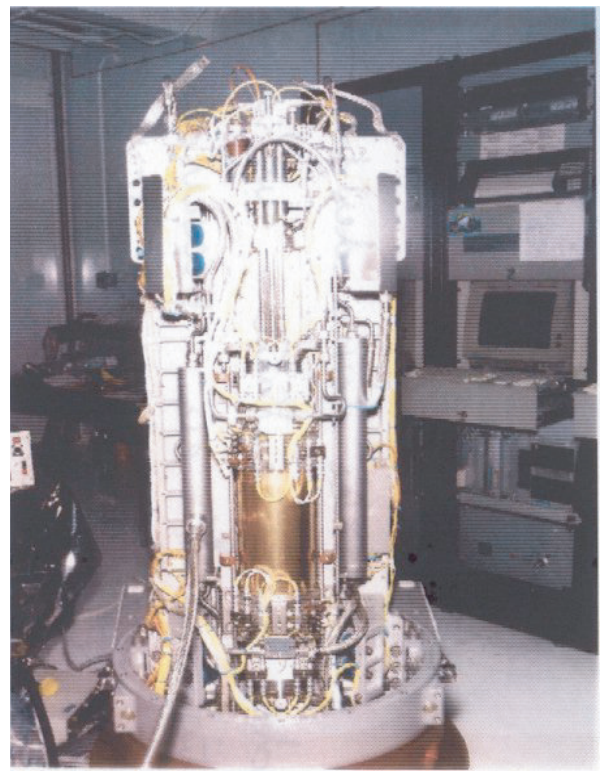

Fig. 2. Développé par le CNES et le Centre d'Études Nucléaires de Grenoble, le four MEPHISTO (Matériel pour l'Étude des Phénomènes Intéressant la Solidification sur Terre et en Orbite). Cet instrument a volé quatre fois entre 1991 et 1997 en fournissant des informations de premier plan sur la déstabilisation des interfaces de solidification d'alliages d'étain en utilisant l'effet Seebeck.

de la Solidification du Centre d'Études Nucléaires de Grenoble (CENG) avec le concours des équipes techniques du Centre National d'Études Spatiales (CNES), la mesure de la tension Seebeck à l'interface de solidification d'un alliage bismuth-étain, rendue très difficile par la nécessité de mesurer de très faibles courants dans un environnement électromagnétique très perturbé, a permis, dans le cadre du projet MEPHISTO (Fig. 2) de localiser pour la première fois le seuil de transition de Mullins et Sekerka entre la morphologie plane et la morphologie cellulaire de l'interface [2].

De même, la possibilité de faire léviter des gouttes de métal en fusion sans contact avec un creuset a permis d'éviter les pollutions mécaniques et chimiques qui sont à l'origine de la solidification. Les expériences faites dans le tube à chute libre du Commissariat à l'Énergie Atomique de Grenoble ont permis d'obtenir le record du monde de surfusion $(900 \mathrm{~K})$ pour le tungstène [3] tandis que cette valeur pour l'ensemble des métaux du tableau périodique a été obtenue récemment et interprétée [4]. De même, des phases métastables A15 dans les alliages rhéniumtungstène ont été découvertes et attribuées à la formation de solutions solides de substitution [5]. La préparation au sol des futures expériences spatiales sur la solidification de matériaux transparents a permis d'obtenir un grand nombre de résultats dont les plus significatifs concernent la dynamique de fronts en lames minces [6] ou l'interaction de la croissance d'échantillons massifs avec la convection [7] (Fig. 3).

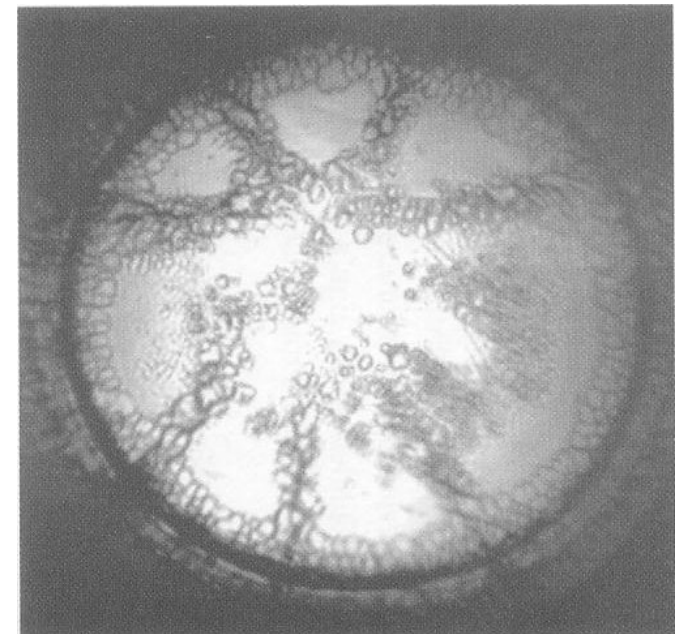

Fig. 3. Image du front de solidification d'un alliage Succinonitrile $-0,2$ wt $\%$ acétone avec une vitesse $V$ de $1,5 \mu \mathrm{m} . \mathrm{s}^{-1}$ dans un gradient thermique $G$ de $30{ }^{\circ} \mathrm{C} / \mathrm{cm}$ dans une configuration « outward hexagonal flow ». Le transport de soluté par la convection localise les transitions structurales de l'interface dans les zones de vitesse très faible. La microgravité, en délocalisant les zones de transition, permettra de les étudier avec précision. Les instabilités sont localisée dans les zones de vitesse convective très faible au lieu d'être réparties de manière homogène et donc observables, comme elles le sont en micropesanteur.

\section{La combustion}

L'intérêt de la micropesanteur dans le domaine de la combustion est la possibilité de faire léviter des gouttelettes de combustible et de leur conserver la symétrie sphérique des études théoriques et de vérifier les théories de bases. De plus lorsque la tension superficielle devient très faible comme lorsqu'on approche des conditions critiques, il n'est plus possible au sol de positionner les gouttelettes sur des supports et la micropesanteur est le seul moyen de réaliser des expériences de combustion. De très nombreuses expériences de ce type ont été réalisées en vols paraboliques d'avion et dans des tours à chute libre dont on pourra trouver une revue dans la référence [8]. Ces expériences se poursuivent aujourd'hui par l'étude de l'évaporation et de la propagation de flammes dans des brouillards de densité homogènes ou dans des assemblées de gouttelettes en interaction, positionnées à l'intersection de fils pour réaliser des réseaux de caractéristiques géométriques diverses. Ces travaux ont tous un grand intérêt appliqué car ils apportent des informations nécessaires à la compréhension de l'allumage et de l'apparition des instabilités des moteurs fusées. Un autre intérêt de la micropesanteur pour la combustion est l'absence de mouvements convectifs et donc de phénomène d'appel d'air. Une bougie en micropesanteur ne prend pas la forme allongée que lui donne au sol la convection mais elle a la forme d'une «méduse » bleutée dont la stabilité est dominée par les échanges thermiques (Fig. 4).

Il devient ainsi possible de souffler sur une surface en combustion avec une vitesse bien inférieure à la vitesse 


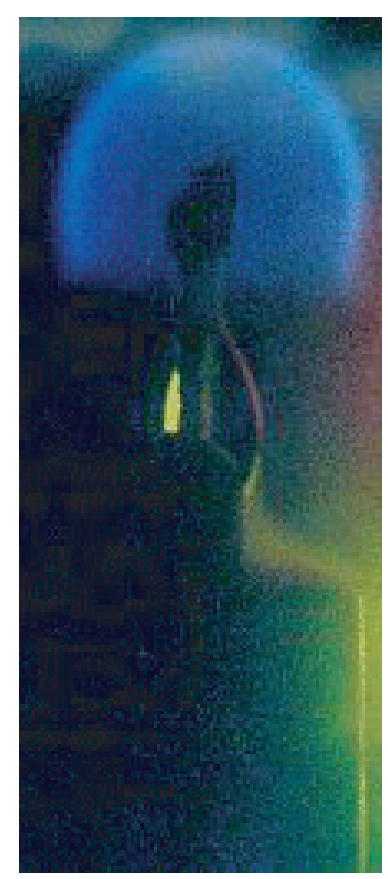

(a)

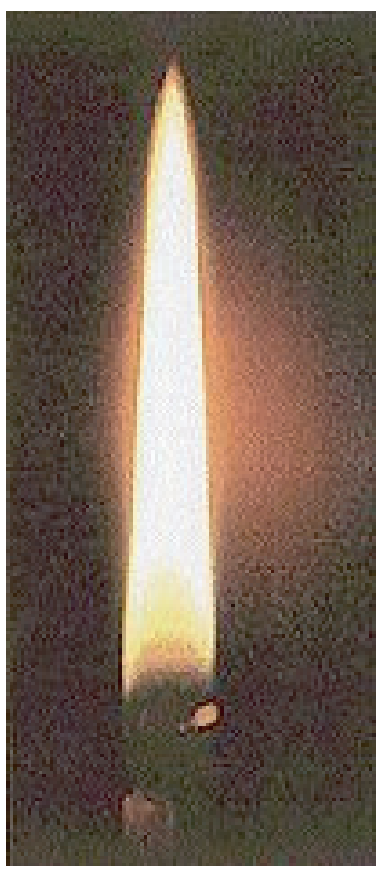

(b)

Fig. 4. Flamme de bougie : en micropesanteur l'absence de mouvement convectif donne à la flamme une géométrie dominée essentiellement par les échanges thermiques (a); au sol, le fort courant convectif thermique qui allonge la flamme vers le haut (b).

imposée au sol par l'appel d'air (la convection) et d'étudier la physique de base dans le cadre d'hypothèses non réalisables au sol (tel le problème d'Emmons pour lequel la vitesse de soufflage peut être faible). La détermination des conditions d'extinction et d'inflammabilité peuvent ainsi aussi contribuer à la sécurité incendie à bord des véhicules orbitaux. Une carte complète des configurations de combustion d'une plaque plane en fonction de la vitesse de soufflage a été obtenue (Fig. 5) en fonction des caractéristiques de la combustion comme la teneur en oxygène de l'atmosphère ambiante. Elle précise dans chacun des régimes de combustion mis en évidence, les rôles respectifs, du rayonnement, lié à la formation des suies, et de la convection forcée. Les projets actuels dans ce domaine s'orientent vers l'étude de la propagation de flammes dans des brouillards à fraction volumique riche en liquide, instables au sol, ou de l'interaction flammevortex dans des poussières constituées de particules dont la taille entraînerait au sol leur sédimentation.

\section{Les interfaces fluides}

Par opposition à la solidification ou à la croissance cristalline qui sont l'étude de l'interface entre un solide et un liquide, les interfaces fluides sont des interfaces entre deux fluides, qu'il s'agisse d'interfaces refermées sur ellesmêmes comme dans le cas des mousses, ou d'interfaces étendues. La micropesanteur permet dans ce domaine d'observer les évolutions sous l'effet du seul transport diffusif dans les phases fluides et des forces de tension de superficielle. Les mousses à forte fraction volumique de liquide, dites mousses humides, ont une durée de vie supérieure en micropesanteur car le drainage gravifique des interfaces n'existe plus et seul le disproportionnement capillaire est encore présent (différences de pression entre des bulles de tailles différentes qui entraîne la vidange de petites bulles dans les grandes et conduit à une augmentation du nombre de grosses bulles). La micropesanteur permet par exemple d'observer la formation des bords de Plateau lors du drainage des interfaces d'une mousse lourde pendant la phase de ressource $(1.8 \mathrm{~g})$ des vols paraboliques de l'avion A300 (Fig. 6).

Quant aux travaux préparatoires au sol sur des mousses sèches, une technique de reconstruction numérique de la mousse à partir d'une tomographie à balayage appliquée à l'étude de la dynamique et de la topologie des mousses sèches tridimensionnelles a permis de montrer que le taux de croissance des bulles est corrélé au nombre de faces. Il s'agit d'une relation universelle qui ne dépend pas de la nature du liquide ou du tensioactif tant que le transport entre les bulles est purement diffusif [9]. La poursuite des recherches dans le domaine des interfaces fluides comporte l'étude des interfaces entre fluides miscibles; ceux-ci posent la question de l'existence d'une tension de surface hors d'équilibre entre deux fluides miscibles qui conduit à des formes d'instabilités associées.

\section{Les objets lévités}

On entend par objets lévités, les objets qui en l'absence de sédimentation restent en suspension dans la phase continue qui les contient, comme les émulsions. Il peut s'agir aussi de matière granulaire, de particules en écoulement ou encore d'écoulements diphasiques en tube. Particulièrement importante pour le développement du transfert thermique avec changement de phase, l'étude des écoulements diphasiques constitue une activité bien établie des recherches en micropesanteur. La diminution considérable de la vitesse relative des phases en micropesanteur entraîne une diminution de la vitesse de glissement et de la turbulence de sillage ainsi que l'augmentation du temps de contact des bulles entre elles. Ceci conduit à une profonde modification de la distribution des bulles dans l'écoulement qui sont principalement situées dans la région centrale. Les modèles théoriques basés sur une équation de bilan prenant en compte l'advection et la coalescence sont bien corrélés avec les expériences et ont donc un caractère prédictif très prometteur [10]. Pour ce qui concerne la matière granulaire, les résultats d'expériences en vols paraboliques d'avion ont permis d'obtenir l'équation d'état d'un gaz granulaire et de montrer l'apparition d'amas compacts dans un gaz de grains vibrés suffisamment dense dont les mécanismes sousjacents pourraient jouer un rôle fondamental dans la formation des anneaux planétaires (Fig. 7) [11,12]. La recherche dans ce domaine s'oriente vers la structuration de 


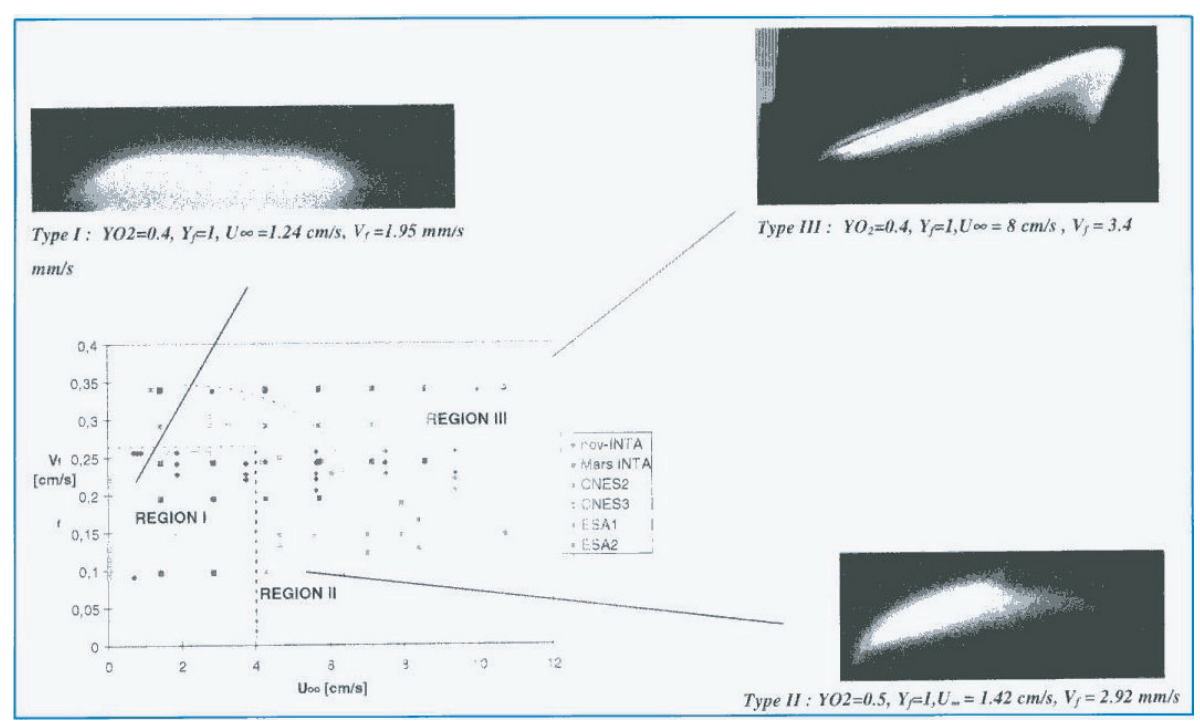

Fig. 5. Forme d'une flamme en fonction de la vitesse de soufflage parallèle à la surface. En permettant des vitesses inférieures à la vitesse dictée par l'appel d'air au sol, l'expérimentation en micropesanteur a permis d'explorer les formes de flammes et d'en étudier les conditions d'extinction, contribuant à déterminer les critères de sécurité incendie à bord des véhicules orbitaux.
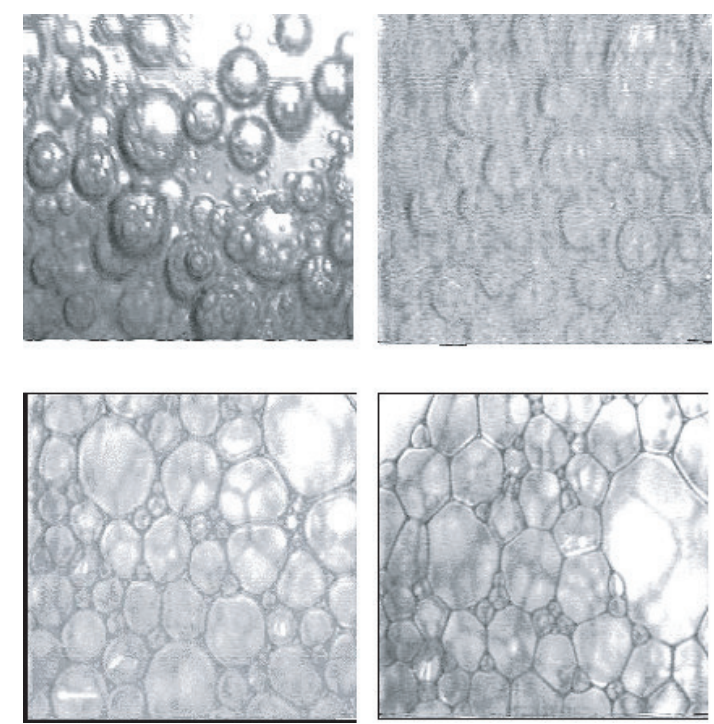

(a)
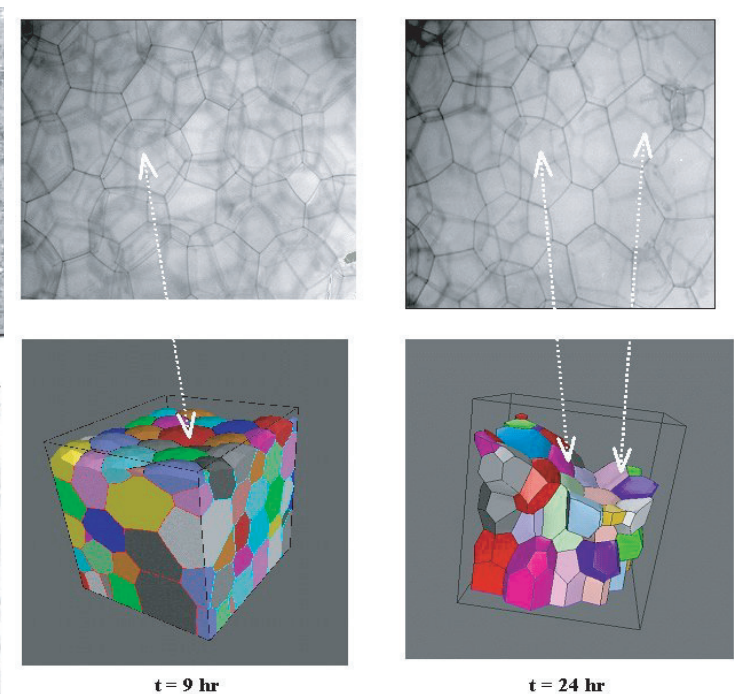

(b)

Fig. 6. (a) Formation des bords de Plateau lors de la ressource au sortir d'une parabole de microgravité, (b) mousse sèche au sol, lors d'expériences préparatoires, et sa reconstitution 3D.

la matière sous l'effet de vibrations contrôlées. La microgravité permet aussi, dans le cadre de projets plus récents, d'étudier et de séparer les effets respectifs des forces de portance hydrodynamiques et de la diffusion hydrodynamique induite par cisaillement sur la séparation des particules polydisperses dans un canal de Hele-Shaw [13]. Enfin, dans le même ordre d'idées mais à une autre échelle, des atomes refroidis par laser dans une mélasse optique en chute libre peuvent être considérés comme des objets lévités dont une large part de la température restante au sol, liée au mouvement de chute libre, est fortement diminuée en micropesanteur. Cette propriété ainsi que la possibilité de lancer des atomes avec une très faible vitesse dans une cavité résonnante a permis d'augmenter d'un facteur dix la précision des horloges atomiques à refroidissement d'atomes par laser. Vérifiée en vols paraboliques en 1997, cette expérience est à l'origine du projet PHARAO qui sera conduit à bord de la Station Spatiale Internationale.

\section{Les fluides critiques}

Les mélanges binaires de fluides sont miscibles en toutes proportions tant que leur température reste supérieure à une certaine valeur. Lorsque la température 


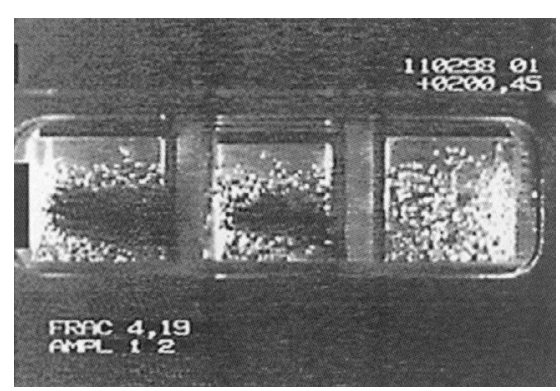

Fig. 7. Matière granulaire vibrée. Lorsque la densité des billes dépasse une certaine valeur, les grains s'agglomèrent pour former un amas, relié aux parois vibrantes par quelques particules qui lui transfèrent l'énergie, au cours d'un processus non complètement expliqué, évocateur d'une transition de phase qui pourrait intervenir dans la formation des anneaux planétaires.

devient inférieure à cette valeur, les phases se séparent et apparaissent alors de très fortes inhomogénéités de densité. En présence de gravité, la phase la plus lourde sédimente dès que les fluctuations de densité atteignent une taille macroscopique et une interface plane se forme rapidement, empêchant toute observation de la croissance des phases. Dans le cas des fluides purs à la transition liquide-gaz, il n'est plus possible, comme dans le cas des mélanges binaires de remplacer par du deutérium l'hydrogène de la phase la plus légère pour égaliser les densités tant elles sont différentes et l'expérimentation en microgravité devient une nécessité. Comme leur compressibilité isotherme diverge au voisinage du point critique liquide-vapeur, le gradient de pression atmosphérique induit une forte stratification, même à l'échelle du laboratoire, comme dans l'atmosphère sur une dizaine de kilomètres. L'homogénéité des échantillons est donc impossible à atteindre au sol même dans des configurations isothermes. Lorsque des perturbations thermiques sont présentes, la très faible diffusivité thermique induit des gradients de température énormes et très localisés, qui induisent les mouvements de convection très intenses et empêchent toute interprétation des expériences. On a pu ainsi étudier en micropesanteur le mouillage apparent d'une paroi par la phase vapeur qui serait dû au recul différentiel de la l'interface provoqué par la production de vapeur à l'interface (Fig. 8). Dans le domaine des transitions de phase, il a été ainsi possible d'étudier sur plusieurs décades la loi de croissance temporelle des fluctuations à la transition liquide gaz dans du $\mathrm{CO}_{2}$ et montrer le rôle clef de la coalescence dans l'existence de deux cinétiques de séparation de phase suivant la valeur initiale de la fraction volumique [14]. Lorsque la fraction volumique est faible, les structures de décomposition de phase croissent par coalescence brownienne selon une cinétique lente proportionnelle à $t^{-1 / 3}$ tandis que lorsque la fraction volumique est supérieure à une valeur voisine de $1 / 3$, les structures croissent par une coalescence hydrodynamique en chaîne qui conduit à une cinétique beaucoup plus rapide en $t^{-1}$. Une relation entre la cinétique et la morphologie a été mise en évidence selon laquelle la cinétique

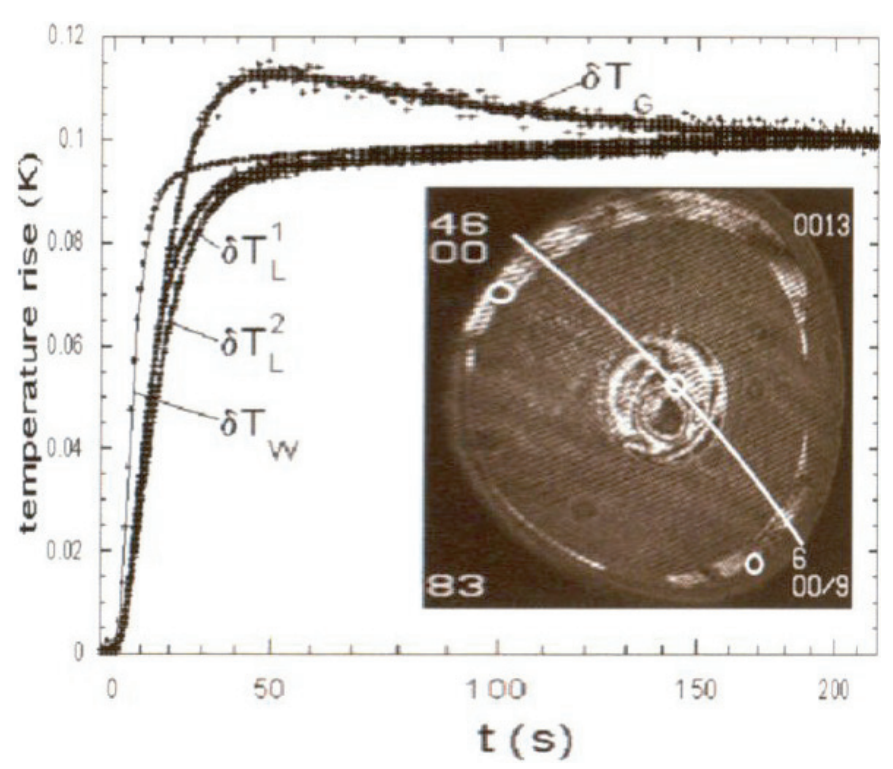

Fig. 8. Surchauffe observée lors d'une trempe thermique de $0,1{ }^{\circ} \mathrm{C}$ dans une cellule de $\mathrm{SF}_{6}$ initialement à $10{ }^{\circ} \mathrm{C}$ sous son point critique : la température du gaz $\left(\delta T_{\mathrm{G}}\right)$ est supérieure à la paroi chauffante $\left(\delta T_{\mathrm{L}}^{1,2}\right)$. La chaleur semble se propager du froid vers le chaud. Le liquide qui mouille la paroi est d'abord chauffé. Comme le gaz est plus dilatable que le liquide l'effet piston dans le gaz est plus important que dans le liquide d'où la température plus élevée.

lente brownienne conserve une structure en gouttelettes tandis que la cinétique rapide correspond à une structure interconnectée.

Dans le domaine des fluides purs, la recherche a été dominée par la découverte d'un quatrième mode de transfert de chaleur, plus rapide que tous les autres connus, qui prend son origine dans la grande compressibilité des fluides supercritiques. Le transfert thermique très rapide observé au sol dans les fluides supercritiques a été dans les années soixante-dix interprété comme d'origine convective, compte tenu de la convection très intense dont ils sont le siège et de leur diffusivité thermique évanescente. La possibilité de supprimer la convection en expérimentant en micropesanteur a permis de vérifier une prédiction théorique [15] selon laquelle un quatrième mode de transfert de chaleur, d'origine thermoacoustique, beaucoup plus rapide que la diffusion, assurerait le transfert de chaleur même en l'absence de convection. Lors du chauffage d'une paroi, le fluide contenu dans la couche très mince de fluide chauffé se dilate et provoque une compression du fluide qui est donc échauffé par transfert thermomécanique. Appelé « effet piston » par la communauté internationale, ce phénomène a été reconnu comme responsable au sol du transfert thermique très rapide attribué au brassage convectif [16]. La micropesanteur a permis en quelque sorte de faire jeter le masque à un phénomène qui se déguisait au sol en convection. Depuis on retrouve sa signature dans des situations qui, sans sa découverte en micropesanteur, seraient restées sans explications ou paradoxales. Les oscillations thermiques au seuil de Rayleigh-Benard [17] ou encore un transfert de 


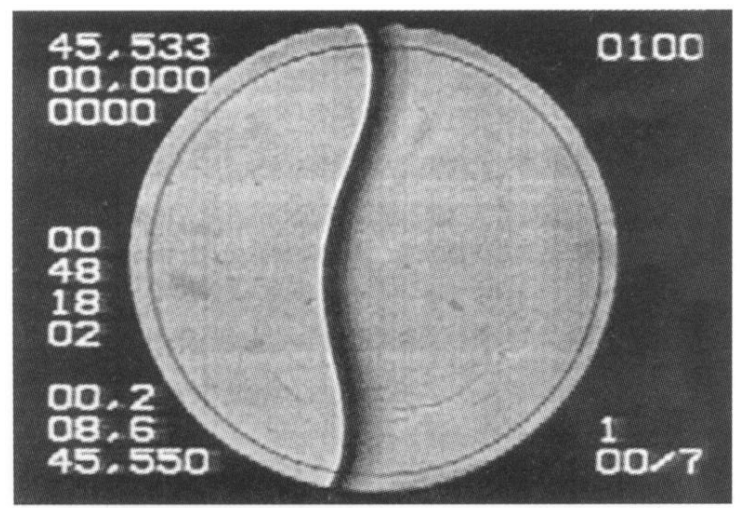

Fig. 9. La courbure de l'interface entre une vapeur et son liquide $\left(\mathrm{SF}_{6}\right)$ s'inverse (b) car l'évaporation fait reculer l'interface, dont la tension de surface est très faible au voisinage du point critique, par transfert de quantité de mouvement. Ce mécanisme a des liens avec l'assèchement d'une paroi au cours de la crise d'ébullition.

chaleur remontant le gradient thermique [18,19] (Fig. 9) en sont de bons exemples. Dans le domaine des fluides critiques, la recherche s'oriente vers la réactivité en phase supercritique, comme l'oxydation, la combustion ou la dissolution.

\section{Les applications de la micropesanteur}

La production industrielle de matériaux aux propriétés physiques améliorées fut, il y a vingt ans, un des éléments qui, avec les vols habités, présida à la mise en place des infrastructures orbitales. Ces recherches ont fait l'objet en grande majorité d'investissements publics, et n'ont pas donné lieu à des opérations commercialement rentables, le prix du lancement et des opérations à bord, même pour des véhicules automatiques et des produits à haute valeur ajoutée, ne permettant pas de dégager des marges suffisantes présentant un intérêt commercial. Par contre des échantillons de matériaux de références ont été obtenus et l'attention de la communauté scientifique a été attirée sur des aspects négligés ou méconnus de la physique des matériaux, comme le rôle du transport convectif sur la sélection du régime de croissance d'un matériaux à partir d'un bain fondu. Les applications industrielles de la micropesanteur se sont alors tournées vers l'obtention d'informations, impossibles à obtenir au sol et d'intérêt pour l'amélioration des procédés industriel terrestres. C'est en ce sens que le programme Microgravity Application Promotion (MAP) de l'Agence Spatiale Européenne (ESA) présente un progrès pour les applications industrielles des recherches en micropesanteur. On peut regretter cependant que la participation financière industrielle soit souvent marginale. Une troisième voie nous paraît prometteuse qui est l'utilisation de l'infrastructure orbitale pour améliorer la gestion des fluides en orbite dont dépend très fortement le bon fonctionnement des systèmes spatiaux. Les recherches sur les problèmes d'interface et de capillarité seront ainsi rapprochées des questions de ballottement et de réservoirs à tension capillaire, de même que les questions de fluides critiques seront appliquées à la gestion des réservoirs de propergols supercritiques et que la dynamique des bulles sera associée aux transferts thermiques par boucle diphasique, tandis que ce qui concerne la dynamique et la rhéologie des mousses sera utile pour l'évaluation précise du reste d'ergol dans les réservoirs en fin de vie des satellites par exemple.

\section{Un exemple de projet futur}

Nous citerons un projet parmi ceux qui nous paraissent les plus représentatifs d'un bon usage de la micropesanteur dans le domaine de la physique des matériaux. Il s'agit d'étudier la structure dynamique du front solideliquide en cours de solidification d'un alliage binaire eutectique transparent. C'est un solide biphasé dont la structure de base est faite de lamelles, c'est-à-dire qu'elle est stationnaire, symétrique, périodique et 1D. Sa transparence permet l'utilisation de méthodes optiques mais il ne peut représenter complètement les alliages métalliques dont il ne reproduit pas la différence de conductivité thermique entre le solide et le liquide. Au sol, pour limiter les effets convectifs perturbateurs, les expériences sont réalisées en échantillons mince; le plan des lamelles reste perpendiculaire aux plans qui définissent la plus petite épaisseur du système. Ces travaux effectués sur l'alliage modèle transparent $\mathrm{CBr}_{4}-\mathrm{C}_{2} \mathrm{Cl}_{6}$ ont permis d'établir la carte des instabilités qui peuvent intervenir en fonction de la vitesse de déplacement du gradient thermique responsable de la solidification et de la concentration de l'alliage. La poursuite de ces travaux sur des échantillons massifs, c'est-à-dire lorsqu'on ne confine plus le système entre deux lames minces, nécessite d'avoir recours à l'expérimentation en micropesanteur pour éliminer la convection. Dans ces conditions, la structure de base sera toujours $1 \mathrm{D}$ mais le front devient un système $2 \mathrm{D}$ et les degrés de liberté dans la troisième direction sont libérés. Il est probable que ces expériences puissent répondre à des interrogations que posent certains eutectiques lamellaires comme par exemple l'origine de la rotation chirale de l'ensemble de la structure.

\section{Conclusion}

La communauté scientifique des utilisateurs des moyens spatiaux d'accès à l'ambiance de micropesanteur utilise ces moyens d'essai avec pertinence. Il est clair que l'expérimentation en micropesanteur constitue aujourd'hui un moyen unique d'observer la matière et de mettre à jour de nouveaux phénomènes qui resteraient sans cela ignorés. Les résultats de ces travaux, dont certains ont été remarqués, sont publiés pour beaucoup d'entre eux dans les grandes revues internationales et constituent une contribution indéniable des recherches en micropesanteur à l'accroissement des connaissances. On note aussi la très 
forte complémentarité de ces recherches avec les questions de gestion des fluides en orbite dont on connaît l'importance pour le développement des nouvelles missions spatiales ou des nouveaux lanceurs et satellites. Cependant, la période écoulée depuis le séminaire de prospective scientifique du CNES tenu à Arcachon au début de 1998 a vu diminuer considérablement le nombre de vols de longue et moyenne durée (comme les vols navette ou capsules automatiques) dans le contexte de l'assemblage d'une station spatiale qui a marqué, et marque toujours le pas au rythme des aléas de la politique américaine. C'est ainsi que nombre de projets majeurs (comme la formation de microstructures lors de la solidification de matériaux transparents ou la physique au voisinage du point critique liquide-gaz), déjà recommandés par le séminaire de prospective d'Arcachon attendent toujours des décisions de développement instrumental ou voient leurs opportunités de vol fuir dans le temps, repoussées de retards en reports. L'impasse dans laquelle pourraient se trouver les grands projets embarquables sur la station spatiale confirme que le débat précédant la décision prise par l'Europe en 1995 de participer au projet de station spatiale n'était pas dénué de pertinence. Il est nécessaire aujourd'hui de conduire une réflexion, avec la communauté scientifique et les grands établissements de recherche, pour définir quels moyens, pourraient à la fois entrer dans un cadre budgétaire adapté à l'ambition des recherches en micropesanteur et assurer l'indépendance de l'Europe.

\section{Références}

[1] R. Bonneville, La micropesanteur, Pour la Science 152 (1990) 102-111

[2] J.J. Favier, P. Lehmann, B. Drevet, J.P. Garandet, D. Camel, S.L. Corriel, A study of stability during directional solidification in microgravity, Lecture Notes in Physics 464 (1995) 77

[3] B. Vinet, L. Cortella, J.J. Favier, P.J. Desré, Highly undercooled $\mathrm{W}$ and Re drops in a ultrahigh vacuum droptube, App. Phys. Lett. 58 (1991) 97-99

[4] B. Vinet, L. Magnusson, H. Fredrikson, Correlations between surface and interface energies with respect to crystal nucleation, J. Coll. Interf. Scie. 255 (2002) 363-374

[5] L. Cortella, B. Vinet, A. Pasturel, P.J. Desré, T. Paxton, M. van Schilfgaarde, Phys. Rev. Lett. 70 (1993) 1469-1472
[6] S. Akamatsu, G. Faivre, Metall. Mater. Trans. 32A (2001) 2039-2048

[7] H. Jamgotchian, N. Bergeon, D. Benieli, P. Vogue, B. Billia, R. Guerin, Localized microstructures induced by fluid flow in directional solidification, Phys. Rev. Lett. 87 (2001) 166105

[8] C. Chauveau,I. Gökalp, D. Segawa, T. Kadota, H. Enomoto, Effects of Reduced Gravity on Methanol Droplet Combustion at High Pressures, Proceedings of the Combustion Institute 28, 2000, pp. 1071-1077

[9] C. Monnereau, M. Vignes-Adler, Dynamics of real three dimensional foams, Phys. Rev. Lett. 80(23) (1998) $5228-5231$

[10] A. Kamp, A.K. Chester, C. Colin, J. Fabre, Bubble coalescence in turbulent flows: a mechanistic model for turbulence induced coalescence applied to bubbly pipe flows under microgravity conditions, Int. J. Multiphase Flow 27 (2001) 1363-1396

[11] E. Falcon, R. Wunenburger, P. Evesque, S. Fauve, C. Chabot, Y. Garrabos, D. Beysens, Cluster formation in a granular medium fluidized by vibrations in low gravity, Phys. Rev. Lett. 83 (1999) 440-443

[12] Vibrating Grains form floating Clumps, Science News 156 (1999)

[13] M. Hoyos, P. Kurowski, Rôle des forces de portance et diffusion hydrodynamique dans les processus de séparation par la technique de SPLITT, J. Phys. IV France 11(Pr6) (2001) Pr6-73

[14] D. Beysens, Y. Garrabos, The phase transition of gazes and liquids, Physica A 281 (2000) 361-380

[15] B. Zappoli, D. Bailly, Y. Garrabos, B. Le Neindre, P. Guenoun, D. Beysens, Anomalous heat transport by the piston effect in supercritical fluids under zero gravity, Phys. Rev. A 41 (1990) 2264-2267

[16] B. Zappoli, S. Amiroudine, P. Carlès, J. Ouazzani, Thermoacoustic and buoyancy-driven transport in square side-heated cavity filled with a near-critical pure fluid, J. Fluid Mech. 316 (1996)

[17] S. Amiroudine, B. Zappoli, Piston effect-induced thermal oscillations at the Rayleigh-Banard threshold in supercritical ${ }^{3} \mathrm{He}$, Phys. Rev. Lett. 90 (2003) 105303

[18] R. Wunenburger, Y. Garrabos, C. Chabot, D. Beysens, J. Hegseth, Thermalization of a two-phase fluid in low gravity: heat transfer from cold to hot, Phys. Rev. Lett. 84 (2000) 4100-4103

[19] Backward heat flow bends the law a bit, Science 288 (2000) 798-791 